ThE ASTROPHYSICAL JouRnAL, 408:L29-L32, 1993 May 1

(C) 1993. The American Astronomical Society. All rights reserved. Printed in U.S.A.

\title{
FIELD COLOR MAGNITUDE DIAGRAM ANALYSIS ALONG THE BULGE MAJOR AXIS ${ }^{1}$
}

\author{
S. ORTOLANi, ${ }^{2}$ E. BiCA, ${ }^{3}$ AND B. BarbuY ${ }^{4}$ \\ Received 1992 September 14 ; accepted 1993 February 11
}

\begin{abstract}
We analyze background fields along the bulge major axis away from the Galactic center by $2.5,13^{\circ}$, and $31^{\circ}$. Our aim is to study the spatial distribution of different stellar populations.

A similar component of young disk main-sequence population is present in all fields. The bulge component prevails in the innermost field, decreases for the $13^{\circ}$ one, and is essentially absent in the $31^{\circ}$ one. Evidence for a weak old metal-poor component is seen in the three fields.

In the $13^{\circ}$ field we detect a peculiar new feature which could be interpreted as an intermediate-age component, and traces of this population in the 2.5 field are also found. This might be an old disk component.

Subject headings: Galaxy: stellar content - Galaxy: structure
\end{abstract}

\section{INTRODUCTION}

Studies of bulge color-magnitude diagrams (CMDs) have so far been performed mostly along the minor axis (Terndrup 1988; Frogel et al. 1990) and further extending to the halo at higher latitudes (Rodgers \& Harding 1989). Such fields have small disk contamination, and the main interest is to study the bulge/halo transition. On the other hand, the major axis provides an opportunity to study in more detail the disk/bulge transition.

In recent years we have been studying CMDs of bulge clusters and accompanying fields: NGC 6553 (Ortolani, Barbuy, \& Bica 1990, hereafter Paper I), NGC 6528 (Ortolani, Bica, \& Barbuy 1992, hereafter Paper II). In this Letter, we analyze a sequence of fields along the bulge major axis, observed in connection with the clusters Terzan 1, NGC 6603, and Lynga 7. The purpose of this study is to identify the relative importance of different stellar populations in these fields.

\section{DATA}

The background fields have been observed in the $V$ and $I$ Cousins filters at the European Southern Observatory (ESO), La Silla (Chile), in a series of observing runs in 1990-1991, with the $1.54 \mathrm{~m}$ Danish telescope.

The RCA CCD No. 5, with $512 \times 32030 \mu \mathrm{m}$ pixel was used. The field of view corresponds on the sky to $4^{\prime} \times 2.5$. The cluster coordinates are listed in Table 1 together with the offset locations of the fields and their Galactic coordinates.

The reductions and calibrations were performed with Midas and DAOPHOT packages, following the standard procedure as already described in Papers I and II.

\section{CMDs AND DISCUSSION}

The projected distribution of the studied fields in Galactic plane coordinates $(l, b)$ is shown in Figure 1 , together with

\footnotetext{
${ }^{1}$ Observations collected at the European Southern Observatory, La Silla, Chile.

${ }^{2}$ Università di Padova, Dept. di Astronomia, Vicolo dell'Osservatorio 5, I-35123 Padova, Italy.

${ }^{3}$ Universidade Federal Rio Grande do Sul, Dept. de Astronomia CP 15051, Porto Alegre 91500, Brazil.

${ }^{4}$ Universidade de São Paulo, IAG, de Astronomia, CP 30627, São Paulo 01051, Brazil.
}

other fields and clusters which we have analyzed in previous papers.

The $V$ versus $(V-I)$ diagrams are shown in Figures 2, 3, and 4 , respectively, for the Terzan $1(l=2.5)$, NGC $6603\left(l=13^{\circ}\right)$, and Lynga $7\left(l=31^{\circ}\right)$ fields.

All the fields contain an extended young disk main-sequence (MS) as expected for such low Galactic latitudes. They also present a red stellar sequence parallel to the young MS, which is identified with evolved stars in the range $100<$ $r t(\mathrm{Myr})<600$ according to classical nonovershooting models, as can be inferred from a comparison with Mermilliod's (1981) CMDs for Galactic open clusters of different ages. The above age, derived from classical models, is reduced by $\sim 30 \%$ in the case of overshooting models (Alongi et al. 1991).

The $l=-31^{\circ}$ field contains a weak old population component, similar to a metal-poor globular cluster, with a red giant branch (Fig. 4) and a group of blue stars which extends to the left of the MS at $V \approx 20.9$ and $(V-I) \approx 1.60$. This component could be identified with the blue horizontal branch (HB) of a moderate to metal-poor old population (inner halo/ thick disk, Gilmore \& Wyse 1985). Assuming $(B-V)_{0}=0$ and $M_{V}=1.0$ for these stars, a distance of $13.8 \mathrm{kpc}$ is derived. This average distance is not unexpected from the halo star distribution in this direction.

The $l=13^{\circ}$ field contains a bulge-rich population which is characterized by a red HB and a red giant branch (RGB). The red $\mathrm{HB}$ is elongated and tilted due to differential reddening and metallicity spread. Evidence of an old metal-poor component, similar to that in the $l=-31^{\circ}$ field, is also present.

An important result is the detection of a concentration of stars at $V \approx 21.0$ and $(V-I) \approx 2.7$, which could be interpreted as an intermediate-age turnoff component. It might be associated with the old disk or a bulge-disk transition subsystem. Further data of this kind would allow one to know better the properties of this component, such as spatial distribution, age, and relative importance with respect to other population components. By observing additional fields along $b$, its scale height could be determined, which would provide important constraints on the collapse model of the Galaxy.

In Terzan 1's field $(l=-2.5)$, the bulge metal-rich component is dominant. We see an RGB typical of very high metallicity (Ortolani, Bica, \& Barbuy 1993, hereafter Paper III). Traces of the intermediate population, detected in the $l=13^{\circ}$ field, might be present (Fig. 2). On the blue side of the MS 


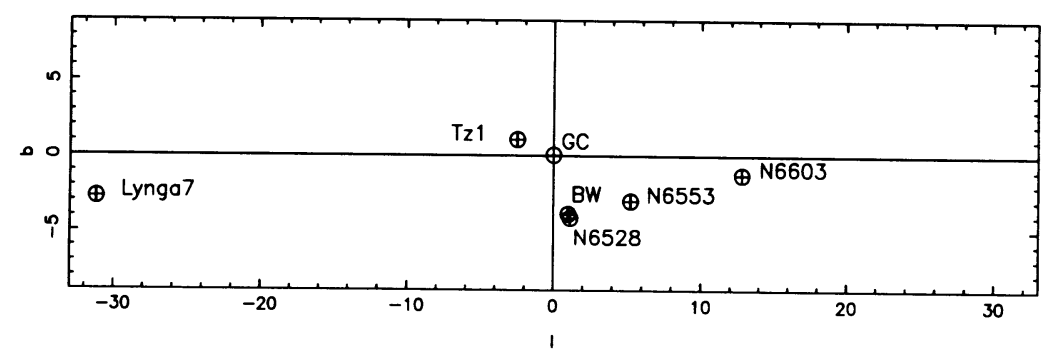

FIG. 1.-Projected distribution of studied fields in Galactic plane coordinates

TABLE 1

CoOrdinates of Studied Background Fields, Where the Cluster COORDINATES AND THE OFFSET LOCATION OF THE FieLDS ARE INDICATED

\begin{tabular}{|c|c|c|c|c|c|}
\hline Cluster & $\alpha_{1950}$ & $\delta_{1950}$ & Offset & $l$ & $b$ \\
\hline Terzan 1 & $17^{\mathrm{h}} 32^{\mathrm{m}} 34^{\mathrm{s}}$ & $-03^{\circ} 00^{\prime} 27^{\prime \prime}$ & $4^{\prime} \mathrm{NW}$ & 357.5 & +1.0 \\
\hline NGC 6602. & 181531 & -182530 & $15^{\prime} \mathrm{N}$ & 12.8 & -1.31 \\
\hline Lynga 7 . & 160704 & -551106 & $20^{\prime} \mathrm{N}$ & 328.8 & -2.8 \\
\hline
\end{tabular}

$(V \approx 22.5)$ there is a weak short sequence, which might correspond to a blue HB of a metal-poor population, or a hot HB component associated with the metal-rich one (Greggio \& Renzini 1990). In fact, with the same assumptions about the blue $\mathrm{HB}$ as above, the derived average distance is $9.1 \mathrm{kpc}$.

The comparison with Baade Window (BW) diagrams by Terndrup (1988) located along the bulge minor axis, at higher Galactic latitude (Fig. 1), shows that the bulge population becomes overwhelming with respect to the blue disk MS. With respect to the possible intermediate-age components detected in our $l=13^{\circ}$ field, it is difficult to judge its presence in Terndrup's BW diagrams.

\section{CONCLUSIONS}

From the study of the three fields observed along the bulge major axis, the following conclusions can be drawn:

1. A well-defined young disk main-sequence population is seen in all fields.

2. The metal-rich bulge component is not present at the $l=-31^{\circ}$ field, appearing at $l=13^{\circ}$ and becoming dominant at $l=-2.5$.

The most important result of this Letter is the presence of a feature which could be identified as an intermediate-age component in the $l=13^{\circ}$ field. Traces of this population seem to appear also in the $l=-2.5$ field. This might be an old disk or a bulge-disk transition component.

Another interpretation is that this possible intermediate-age

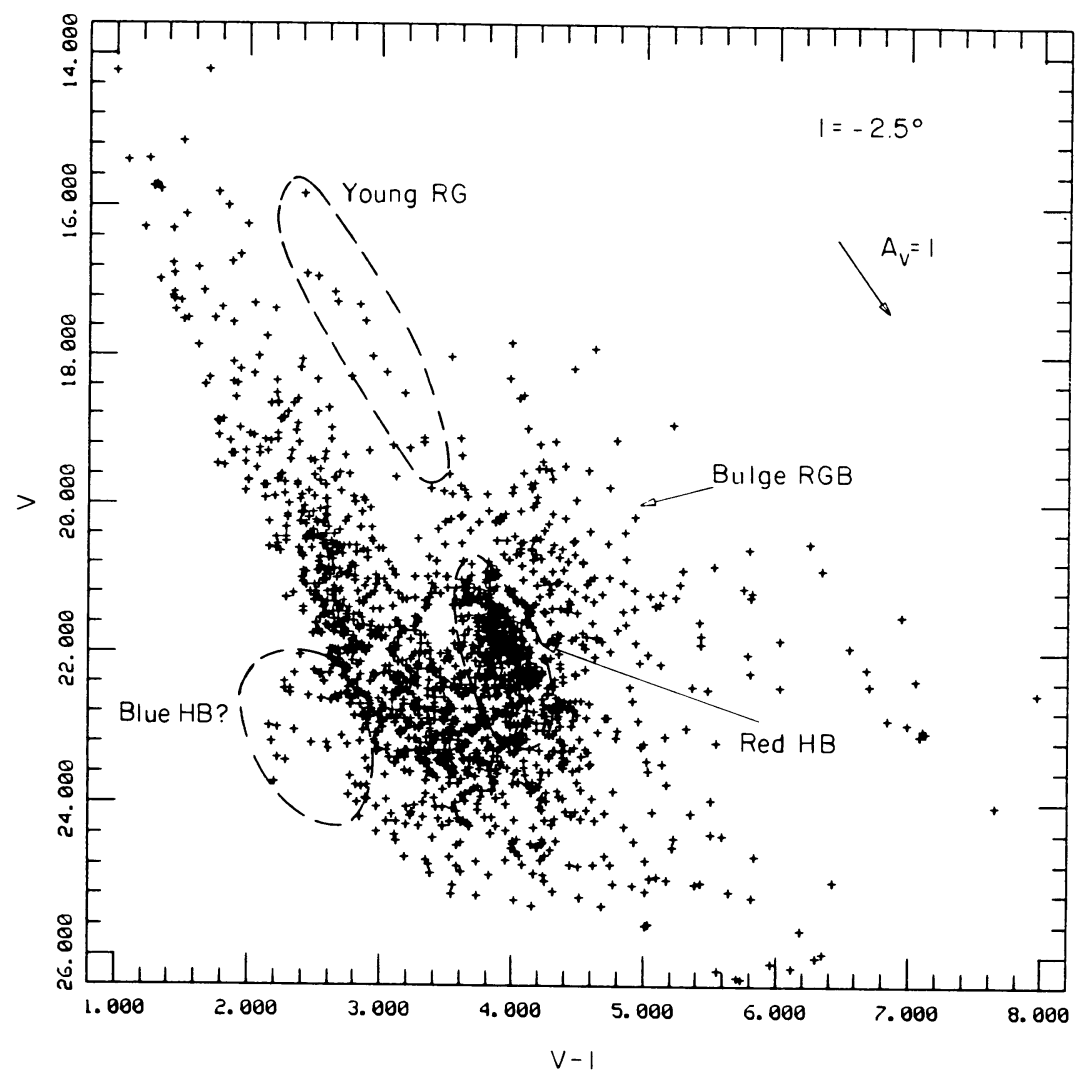

Fig. 2. $-V$ vs. $(V-I)$ background field of Terzan 1, where identified stellar sequences are labeled 


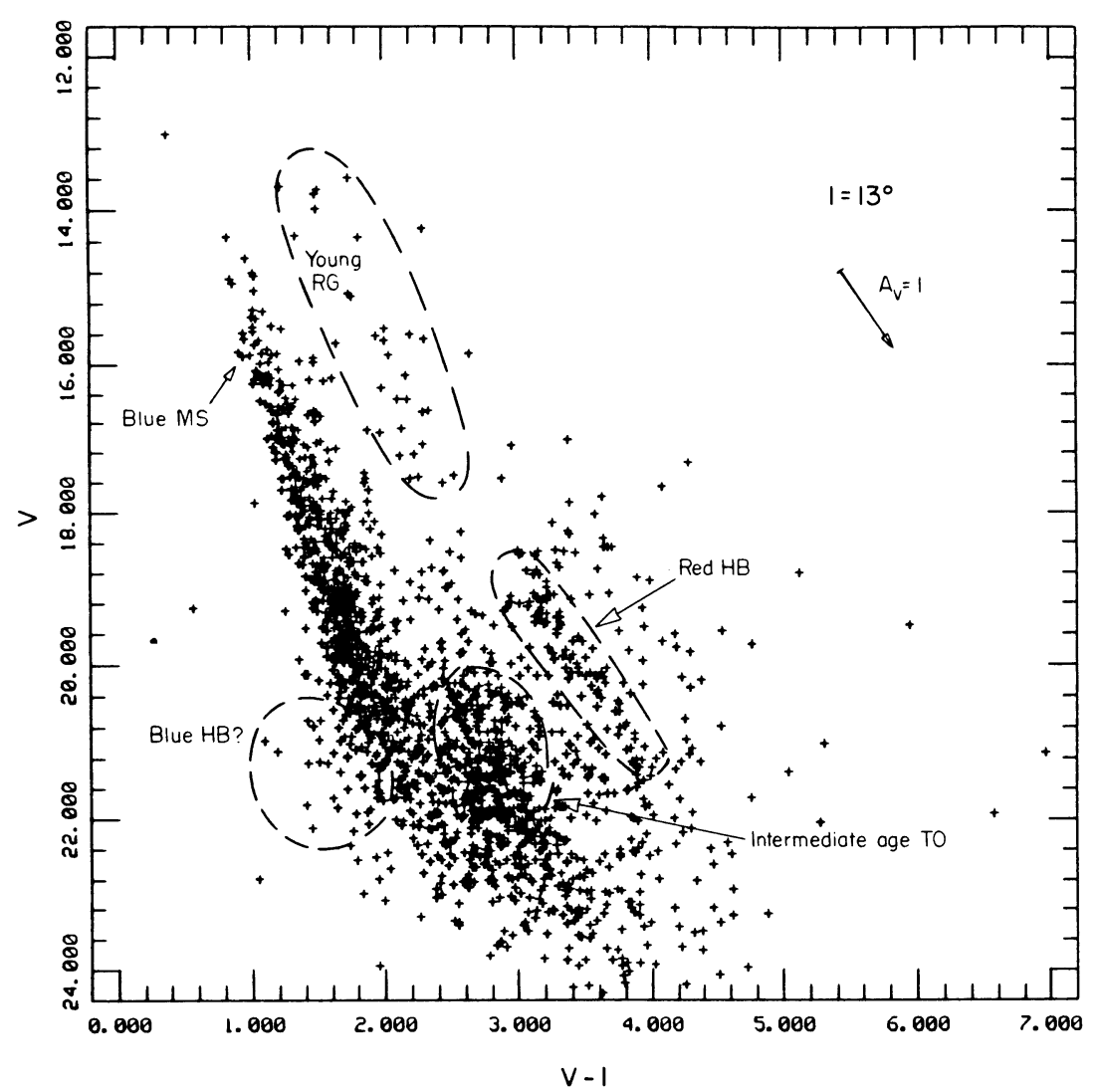

Fig. 3. $-V$ vs. $(V-I)$ background field of NGC 6603

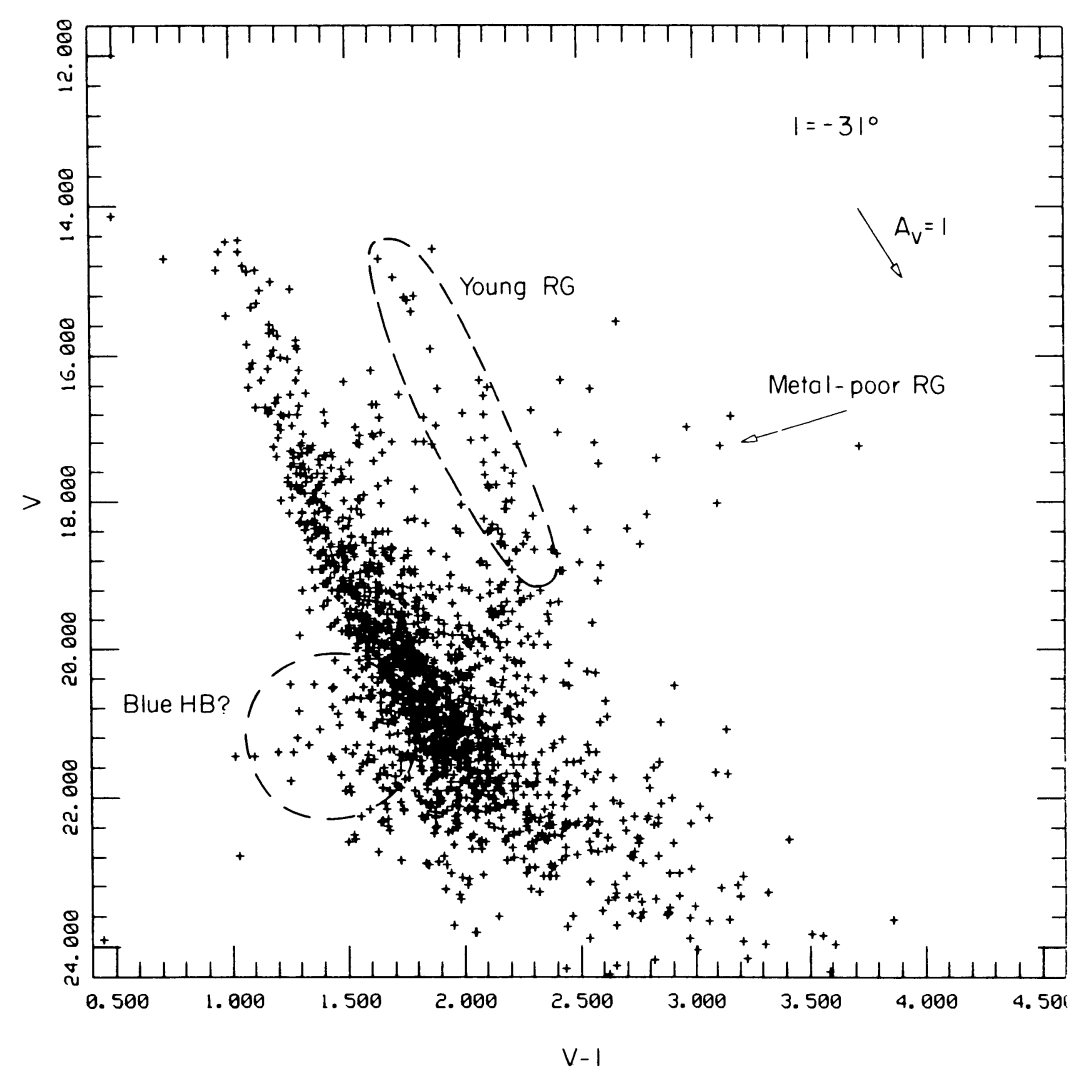

FIG. 4. $-V$ vs. $(V-I)$ background field of Lynga 7 
component is part of a bar system in the central parts of the Galaxy (Blitz \& Spergel 1991). Indeed, a large fraction of bar stellar populations in the LMC are of intermediate age. Since the age of the identified turnoff component would be 5-10 Gyr, this age is consistent with an intermediate age of the bar (Frogel \& Blanco 1990; Bica, Clariá, \& Dottori 1992; Hardy et al. 1984). Further evidence for a coincidence with the bar is its location where evidence for the presence of a bar has been detected: these are IRAS asymptotic giant branch stars and Miras, located at low latitudes and longitudes of $+15^{\circ}$ (de Zeeuw 1992).

An alternative interpretation is an association with a large star cloud in Sagittarius, where the field is projected. In fact, Bica, Ortolani, \& Barbuy (1993) detected a range of ages in the star cloud of at least 100-600 Myr, but it could extend further up to intermediate ages.
Alongi, M., Bertelli, G., Bressan, A., \& Chiosi, C. 1991, A\&A, 244, 95

Bica, E., Clariá, J. J., \& Dottori, H. 1992, AJ, 103, 1859

Bica, E., Ortolani, S., \& Barbuy, B. 1993, A\&A, in press

Blitz, L., \& Spergel, D. N. 1991, ApJ, 379, 631

de Zeeuw, T. 1992, in IAU Symp 148, The Stellar Populations of Galaxies, ed. B. Barbuy \& R. Renzini (Dordrecht: Kluwer) 51

Frogel, J. A., \& Blanco, V. M. 1990, ApJ, 365, 168

Frogel, J. A., Terndrup, D. M., Blanco, V. M., \& Whitford, A. E. 1990, ApJ, 353,494

Gilmore, G., \& Wyse, R. F. G. 1985, AJ, 90, 2015

\section{REFERENCES}

Greggio, L., \& Renzini, A. 1990, ApJ, 364, 35

Hardy, E., Buonanno, R., Corsi, C. F., Janes, K. A., \& Schommer, R. A. 1984, ApJ, 278, 592

Mermilliod, J. C. 1981, A\&AS, 44, 467

Ortolani, S., Barbuy, B., \& Bica, E. 1990, A\&A, 236, 362 (Paper I)

Ortolani, S., Bica, E., \& Barbuy, B. 1992, A\&AS, 92, 441 (Paper II)

. 1993, A\&A, 267, 66 (Paper III)

Rodgers, A. W., \& Harding, P. 1989, AJ, 97, 1036

Terndrup, D. M. 1988, AJ, 96, 884 